\title{
Characteristics of a prevalent vertebral deformity predict subsequent vertebral fracture: results from the European Prospective Osteoporosis Study (EPOS)
}

\author{
Mark Lunt, ${ }^{\mathrm{a}, *}$ Terence W. O’Neill, ${ }^{\mathrm{a}}$ Dieter Felsenberg, ${ }^{\mathrm{b}}$ Jonathan Reeve, ${ }^{\mathrm{c}}$ John A. Kanis, ${ }^{\mathrm{d}}$ \\ Cyrus Cooper, ${ }^{\mathrm{e}}$ Alan J. Silman, ${ }^{\mathrm{a}}$ and the European Prospective Osteoporosis Study Group ${ }^{1}$ \\ ${ }^{a}$ ARC Epidemiology Unit, University of Manchester, Manchester, M13 9PT, UK \\ ${ }^{\mathrm{b}}$ Department of Radiology and Nuclear Medicine, Free University, Berlin, Germany \\ ${ }^{\mathrm{c}}$ Strangeways Research Laboratory, Cambridge, CB1 8RN, UK \\ ${ }^{\mathrm{d}}$ Centre for Metabolic Bone Disease, Sheffield, S10 2RX, UK \\ ${ }^{\mathrm{e}}$ MRC Environmental Epidemiology Unit, University of Southampton, Southampton, SO9 4XY, UK
}

Received 10 April 2003; revised 16 June 2003; accepted 27 June 2003

\begin{abstract}
The presence of a prevalent vertebral deformity increases the risk of a future vertebral fracture. The aim of this study was to determine whether certain characteristics of the prevalent deformity, including its shape and location in the spine, influenced this effect. The 3100 men and 3500 women who took part in this analysis were recruited from population registers for participation in the European Prospective Osteoporosis Study (EPOS). Subjects had lateral thoracic and lumbar spine x-rays at baseline, and again after a mean interval of 3.8 years. Prevalent morphometric vertebral deformities on the baseline film were identified by the McCloskey-Kanis method. Incident fractures were defined as vertebrae that also satisfied the McCloskey-Kanis criterion for prevalent deformities on the follow-up film, and in addition had at least one height (anterior, mid, or posterior) which had reduced by at least $20 \%$ between films. Poisson regression was used to assess the association between various characteristics of the prevalent deformity and the risk of an incident vertebral fracture, with generalised estimating equations used to allow for the fact that each subject contributed several vertebrae to the analysis. The risk of an incident fracture increased with the number of prevalent deformities: relative risk (RR) for one prevalent deformity 3.2 (95\% confidence interval (CI); 2.1, 4.8); 9.8 (95\% CI;6.1, 15.8) for 2; and 23.3 (95\% CI;15.3, 35.4) for 3 or more. Relative risks differed significantly according to the shape of the prevalent deformity, ranging from 5.9 (95\% CI; 4.1, 8.6) if the anterior and mid heights were reduced to $1.6(95 \% \mathrm{CI} ; 0.8,3.2)$ if the posterior and mid heights were reduced. Risks varied also according to the severity of the deformity. There were fivefold differences in relative risk of incident fracture depending on the location of the prevalent deformity within the spine. Compared to vertebrae in subjects with no deformities at baseline, the relative risk of an incident fracture within three vertebrae of a prevalent deformity was greater $(7.7$ (95\% CI;5.6, 10.5)) than the risk in more distant vertebrae $(4.0(95 \% \mathrm{CI} ; 2.6,6.0))$. In summary, the risk of a subsequent vertebral fracture in individuals with preexisting deformities is importantly influenced by the characteristics of these deformities. (C) 2003 Elsevier Inc. All rights reserved.
\end{abstract}

Keywords: Osteoporosis; Vertebral deformity; Incident vertebral fracture; Prediction; Prospective study; Population study

\footnotetext{
* Corresponding author. ARC Epidemiology Unit, University of Manchester, Stopford Building, Oxford Road, Manchester, M13 9PT, UK. Fax: +00-44-161-275-5043.

E-mail address: Mark.Lunt@man.ac.uk (M. Lunt).

${ }^{1}$ The EPOS Study Group: Project Coordination: J. Reeve (Institute of Public Health, Cambridge, UK); A. Silman (ARC Epidemiology Unit, University of Manchester, UK). Radiology Group: D. Felsenberg (coordinator), G. Armbrecht, and W. Gowin (Freie University Berlin, Germany). Data Management Group: W. Cockerill, J.D. Finn, A.A. Ismail, M. Lunt, T.W. O’Neill, and S. Pye (ARC Epidemiology Unit, University of Manchester, UK); C. Matthis and H.H. Raspe (Institute for Social Medicine, Lubeck, Germany). Principal Investigators in Participating Centres: D. Banzer (Berlin, Germany); L.I. Benevolenskaya (Moscow, Russia); A. Bhalla (Bath, UK); J.B. Cannata (Oviedo, Spain); J. Dequeker (Leuven, Belgium); R. Eastell (Sheffield, UK); B. Felsch (Jena, Germany); J. Franke (Erfurt, Germany); C. Gennari (Siena, Italy); S. Havelka (Prague, Czech Republic); K. Hoszowski (Warsaw, Poland); I. Jajic (Zagreb, Croatia); J. Janott (Bochum); O. Johnell (Malmo, Sweden); A. Lopes Vaz (Oporto, Portugal); R. Lorenc (Warsaw, Poland); G. Lyritis (Athens, Greece); P. Masaryk (Piestany, Slovakia); C. Matthis (Lubeck, Germany); T. Miazgowski (Szczecin, Poland); H.A.P. Pols (Rotterdam, Netherlands); G. Poor (Budapest, Hungary); H.H. Raspe (Lubeck, Germany); J. Reeve (Cambridge, UK); D.M. Reid (Aberdeen, UK); W. Reisinger (Berlin, Germany); C. Scheidt-Nave (Heidelberg, Germany); J.J. Stepan (Prague, Czech Republic); C.J. Todd (Cambridge, UK); K. Weber (Graz, Austria); A.D. Woolf (Truro, UK).
} 


\section{Introduction}

Ascertainment of low trauma fracture is a marker for future fracture risk elsewhere, presumably through susceptibility via increased bone fragility [1]. As an example, vertebral fracture is strongly associated with the future risk not only of further vertebral fracture [2] but also of limb fracture [3]. The explanation for these associations is only partly through reduced BMD [1,4]. Mechanical influences resulting from the initial deformity [2] and other factors reflecting bone "quality," which are difficult to quantify noninvasively, may also be important [5].

Vertebral deformity is the most frequent type of osteoporotic fracture and is frequently not reported by the patient to a physician [6] so is underrepresented in clinical databases [7]. There are, therefore, a number of unanswered questions relating to future vertebral fracture risk following the ascertainment of a prevalent deformity. The first question is whether the risk of a new vertebral fracture varies by the vertebral level of the preexisting deformity. If so, this may reflect the fact that at some vertebral levels a prevalent deformity may be more likely to reflect generalised osteoporosis. Second, is the risk of a new fracture greater in vertebrae lying close to the level of the initial fracture? If so, this may reflect the importance of mechanical influences of a prevalent deformity impacting more on adjacent vertebrae. The third question is whether the shape of the prevalent deformity (wedge, concavity, or crush [8]) influences the risk of subsequent fracture. In previous work, the association between BMD and the presence of prevalent deformity differed between deformities of different shapes [4]. Finally, if any of these characteristics do affect vertebral fracture incidence, it is unknown whether these effects are the same in men as in women.

We have explored these questions using data from the European Prospective Osteoporosis Study (EPOS).

\section{Methods}

\section{Design}

EPOS is a multicentre population-based prospective study. At baseline, subjects had a lateral spinal radiograph to identify those with a prevalent deformity [9]. After a mean follow-up of 3.8 years, a second radiograph of each subject was taken, to identify incident vertebral fractures [10].

\section{Baseline survey}

The subjects who took part in the analysis were recruited from population registers for participation in a prospective study of osteoporosis - the European Vertebral Osteoporosis Study (EVOS) and its subsequent prospective phase (EPOS). Detailed methods have been described elsewhere
[9]. In brief, at baseline, men and women aged 50 years and over were recruited from population registers in 36 European centres. Stratified sampling was used with the aim of recruiting equal numbers of men and women in each of six 5-year age bands from 50-54 to 75-79 years.

Baseline radiographs were taken according to a standard written protocol which included details concerning positioning of subjects and radiographic technique. The thoracic film was centered at T7 and the lumbar film at L2. Radiographs were taken with the patient in the left lateral position and, for the thoracic films, the breathing technique was used to blur overlying structures not related to the spine.

All radiographs were evaluated morphometrically in Berlin by one of three observers, using a mouse-caliper system on a backlit digitising board. The senior radiologist (DF) trained all three observers. Six points were marked on each of the 13 vertebral bodies from T4 to L4 to describe vertebral shape. All points were chosen as accurately as possible. They were positioned on the outer edge of the upper and lower endplates. Both anterior measurement points were placed in the anterior fifth (the vertebra was divided into five parts in the anterior-posterior direction) of the vertebra when degenerative changes showed spondlylophytes, sclerotic endplate thickening, or anterior edge "lipping." When no degenerative changes were present, the anterior points were placed at the intersections of the upper and lower endplate with a line drawn tangential to the anterior edge and perpendicular to a midvertebral line. The medial measurement points were placed at positions which accounted for projection artifacts. In the event of apparent rotation of the vertebra, with two lines projected, the central point between projection lines was chosen. They were positioned in the central 3/5th. The posterior measurement points were positioned at the posterior endpoints of the upper and lower endplates. The coordinates were recorded on to an electronic grid and subsequently stored on a VAX workstation.

Using these six points, the anterior (A), mid (M), and posterior $(\mathrm{P})$ heights were determined for each vertebral body in millimetres. The McCloskey-Kanis algorithm was used to identify prevalent deformities [11]. This algorithm classifies deformed vertebrae as one of three shapes: "biconcave," "wedged," or "crushed." However, it has previously been shown that it is possible to further subdivide the shapes defined by this algorithm, based on which combinations of the three heights are reduced [4]. The relationship between the McCloskey-Kanis shapes and the more detailed classification is shown in Table 1. This is of interest since bone mineral density (BMD), for example, is more strongly associated with some subtypes than others [4].

\section{Follow-up survey}

Of the original participating centres, 29 invited subjects who had taken part in the baseline survey to return for a second $\mathrm{x}$-ray. The mean interval between radiographs was 
Table 1

Shapes of morphometrically defined deformities

\begin{tabular}{|c|c|c|c|c|}
\hline \multicolumn{3}{|c|}{ Vertebral height } & \multicolumn{2}{|l|}{ Classification } \\
\hline Anterior (A) & Mid (M) & Posterior $(\mathrm{P})$ & McCloskey-Kanis ${ }^{\mathrm{a}}$ & Detailed \\
\hline Normal & Normal & Normal & Normal & Normal \\
\hline Normal & Normal & Reduced & Normal & Normal \\
\hline Normal & Reduced & Normal & Biconcavity & M only \\
\hline Reduced & Normal & Normal & Wedge & A only \\
\hline Reduced & Reduced & Normal & Wedge & $\mathrm{A}+\mathrm{M}$ \\
\hline Normal & Reduced & Reduced & Wedge & $\mathrm{M}+\mathrm{P}$ \\
\hline Reduced & Normal & Reduced & Crush & $\mathrm{A}+\mathrm{P}$ \\
\hline Reduced & Reduced & Reduced & Crush & $\mathrm{A}+\mathrm{M}+\mathrm{P}$ \\
\hline
\end{tabular}

${ }^{\mathrm{a}}$ Reference [11].

3.8 years, but the mean interval varied considerably between centres, from 1.9 to 5.4 years. Of the 13,633 subjects with a baseline radiograph in the 29 participating centres, 6667 returned for a follow-up.

\section{Follow-up radiographs}

The follow-up radiographs were taken according to the same standard protocol. They were also evaluated morphometrically in Berlin, by a single observer, using exactly the same methodology as the baseline radiographs. This observer was not one of the three observers who made the baseline measurements, but was also trained by the senior radiologist (DF).

Then all film pairs were reviewed side-by-side on the same screen with image enhancement (magnification, contrast) by the senior radiologist who made a clinical judgement as to whether there had been a fracture event in any vertebral body between the two films [10]. By comparing the recorded height ratios to the image of the initial film, the senior radiologist could determine whether a gross error in point placement had occurred, and if necessary remeasure all vertebral heights. All vertebrae considered to be outside the criteria defining them as definitely normal (one of the vertebral height ratios $(\mathrm{A} / \mathrm{P}$ or $\mathrm{M} / \mathrm{P})$ was less than 0.75 or there was a change of 0.15 or more in any vertebral height ratio between the two films) were measured twice to ensure that measured changes between films recorded in the database were based on real changes of shape and were not the result of measurement errors. The software used in these side-by-side comparisons recorded changes in height ratios. Following these measurement procedures, adjustments were made to the measurements derived from each pair of films (pre, post) to bring each pair of measurements made on a particular vertebral body to the same magnification. The procedure used [12] was based on the principle that all vertebrae judged nonfractured should not have changed their vertebral heights, which would only therefore on average differ between films due to magnification differences arising from differences in technique. This correction procedure in turn allowed changes in measured vertebral heights to be calculated arithmetically from the ratio changes. Our approach was modelled in part on our successful pilot study [13].

A vertebra was classified morphometrically as an incident fracture on the follow-up film if it:

(i) satisfied the McCloskey-Kanis algorithm on the follow-up film; and

(ii) at least one height had been reduced by $20 \%$ on the follow-up compared to the baseline film, and this reduction was at least $4 \mathrm{~mm} \mathrm{[14].}$

\section{Statistical methods}

The following characteristics of any baseline prevalent deformity, all measured at baseline, were used as predictors of incident fracture: (i) the presence of at least one deformity in the spine, (ii) the number of deformities in the spine, (iii) the presence of a deformity at each individual vertebral level, (iv) the presence of at least one deformity of a given shape (using both the McCloskey-Kanis and more detailed classifications), (v) the severity of the baseline deformity, and (vi) the presence of a deformity in the vertebra directly above, directly below, or within three vertebrae above or below.

The probability of incident fracture (any shape) at follow-up was predicted in each vertebra individually, based on age, gender, and vertebral level, as well as the predictors described above. Poisson regression was used since it allows for different lengths of follow-up in different individuals.

In this analysis, each individual contributed up to 13 vertebrae. Conventional generalised linear models assume that all observations are independent, and so they could not be used in this case. Instead, when data are "clustered" in this way, generalised estimating equations can be used to model the within-cluster correlation and hence obtain unbiased estimates [15]. The procedure "xtgee" in Stata [16] was used for this purpose. Although it is possible for a vertebra to be classed as both a prevalent deformity and an incident fracture (if there was a deformity present in the baseline film and that vertebra showed a substantial change in shape between the two radiographs) this was rare, and only vertebrae classed as normal on the baseline radiograph were included in the model.

A baseline model containing age, sex, and vertebral level was fitted first, since all of these factors were expected to influence the probability of an incident fracture. Then each variable was added in turn and the fit of the model assessed using the Akaike Information Criterion (AIC) [17]. The AIC of a given model is defined as

$$
\mathrm{AIC}=-2 \log (L)+2 P,
$$

where $\log (L)$ is the $\log$ likelihood of the model and $P$ is the number of parameters in the model. Using the AIC is very similar to comparing log likelihoods to assess the fit of a model, but unlike comparing log likelihoods, it is not re- 
Table 2

Location of baseline vertebral deformities

\begin{tabular}{lc}
\hline Vertebral level & Total number \\
\hline T4 & 34 \\
T5 & 63 \\
T6 & 79 \\
T7 & 106 \\
T8 & 102 \\
T9 & 74 \\
T10 & 44 \\
T11 & 76 \\
T12 & 109 \\
L1 & 135 \\
L2 & 82 \\
L3 & 65 \\
L4 & 39 \\
& \\
& \\
\hline
\end{tabular}

stricted to nested models (models are said to be nested if one model can be obtained from the other by adding one or more variables). The model with the smallest AIC is the best fitting.

The area under the receiver operating curve [18] (AUC) was also used to compare models. This represents the probability that, given a randomly selected subject with an incident fracture and a randomly selected subject without an incident fracture, the predicted risk of deformity from the model will be greater for the subject with a deformity than for the subject without.

\section{Results}

\section{Population characteristics}

Of 6364 men and 7269 women who had an initial radiograph in the 29 centres taking part in EPOS, 3161 men and 3506 women returned for a second radiograph.

Overall, $10.2 \%$ of both the men and the women who were $\mathrm{X}$-rayed twice had one or more baseline deformities. There were a total of baseline 1008 deformities in the 76,185 vertebrae examined, with 495 subjects having a single deformity, 116 having 2, 33 having 3 , and 35 having 4 or more. The distributions of the locations of these deformities are given in Table 2. Deformities were most common around T7-T8 and T12-L1 [19]. The numbers of the different types of deformities, using both the McCloskeyKanis classification and the more detailed classification, are given in Table 3. The most common type of deformity was biconcavity, accounting for more than a third of all deformities.

Influence of age, gender, and vertebral level on fracture incidence

Follow-up evaluation of the 75,177 vertebrae that were not deformed at baseline revealed 84 incident deformities in
63 men and 195 in 135 women. This gives a total fracture incidence of 5.9 fractures per 1000 person/years in men (95\% CI: 4.6, 7.4) and 10.1 fractures per 1000 person/years in women (95\% CI: 8.5, 11.9).

The relative risk increased by a factor of 2.1 per decade increase in age $(95 \%$ CI $1.7,2.5)$ and was 2.3 times greater in women than in men $(95 \%$ CI 1.6, 3.4). The relative increase with age did not differ between men and women $(P$ $=0.4)$.

After adjusting for age and sex, the incidence of vertebral fracture differed at different vertebral levels $\left(\chi^{2}=78.7\right.$ on 12 d.f., $P<0.0001$ ), although there was no evidence that this distribution differed between men and women $\left(\chi^{2}=9.1\right.$ on 12 d.f., $P=0.7)$. The predicted probabilities of an incident fracture in each vertebra of a woman aged 65 are given in the Appendix: the probabilities for men and for other ages can be calculated as explained in the Appendix.

Because of the important natural variation in risk of an incident fracture by age, gender, and vertebral level, subsequent analyses were adjusted for these variables.

Influence of the characteristics of baseline deformities on fracture incidence

\section{Number of baseline deformities}

After adjusting for age, gender, and vertebral location, vertebrae in subjects with a morphometrically defined deformity at baseline were 6.1 times as likely to suffer an incident fracture as those in subjects without a baseline deformity $(95 \% \mathrm{CI} ; 4.5,8.2)$. The risk increased with the number of deformities at baseline, being 3.2 (95\% CI; 2.1, 4.8) for those with a single deformity, 9.8 (95\% CI; 6.1, 15.8) for those with two, and 23.3 (95\% CI; 15.3, 35.4) for those with three or more. There was no difference in the strength of the association between men and women $\left(\chi^{2}=\right.$ 0.2 on 3 d.f., $P>0.9$ ).

\section{Location of baseline deformity}

The risk of a subsequent incident fracture varied according to the location of the baseline deformity. The relative risk of incident fracture according to the level of the prevalent deformity at baseline is given in Table 4 . The risk of

Table 3

Numbers of baseline deformities according to vertebral shape using McCloskey-Kanis method and detailed classification (see Table 1)

\begin{tabular}{llc}
\hline Classification of baseline deformity & & Number \\
\hline McCloskey-Kanis & Detailed & \\
\hline Biconcavity & M only & 396 \\
& A only & 152 \\
Wedge & A + M & 270 \\
& P+M & 84 \\
Crush & A + P & 17 \\
& A $+M+P$ & 89 \\
\hline
\end{tabular}


Table 4

Relative risks of incident vertebral fracture according to level of baseline deformity

\begin{tabular}{ll}
\hline Vertebra & Relative risk $(95 \% \mathrm{CI})^{\mathrm{a}}$ \\
\hline T4 & $0.8(0.3,2.6)$ \\
T5 & $4.6(2.5,8.2)$ \\
T6 & $2.9(1.6,5.3)$ \\
T7 & $2.3(1.3,4.2)$ \\
T8 & $1.8(0.9,3.4)$ \\
T9 & $1.2(0.5,2.5)$ \\
T10 & $1.3(0.5,3.1)$ \\
T11 & $1.2(0.6,2.6)$ \\
T12 & $1.8(0.9,3.7)$ \\
L1 & $2.5(1.4,4.4)$ \\
L2 & $2.1(1.0,4.7)$ \\
L3 & $2.4(1.0,5.5)$ \\
L4 & $0.8(0.3,2.3)$ \\
\hline
\end{tabular}

${ }^{\text {a }}$ Relative risk for an incident fracture at any level associated with a prevalent deformity at the given level.

a subsequent fracture was greatest for prevalent deformities at T5-T7 and L1-L3. It is interesting that although the vertebrae in which prevalent deformities are most common are associated with an increased risk of incident fracture, some vertebrae in which prevalent deformities are comparatively rare (e.g., T5, L3) are also associated with substantial increases in risk. Again, the relative risks did not differ significantly between men and women $\left(\chi^{2}=12.6\right.$ on 11 d.f., $P=0.3$ ).

\section{Shape of baseline deformity}

Using the McCloskey-Kanis classification of shapes (wedge, biconcavity, and crush), the relative risk of an incident fracture did not differ significantly according to shape ( $\chi^{2}=0.6$ on 2 d.f., $P>0.5$ ). However, using the finer morphometric distinctions in shape (Table 1), there were statistically significant differences between the relative risks for the different morphometric shapes of deformity $\left(\chi^{2}=\right.$ 15.8 on 5 d.f., $P=0.008$ ), seen in Table 5 . There was no increased risk of incident fracture for subjects with either $\mathrm{A}+\mathrm{P}$ or $\mathrm{M}+\mathrm{P}$ prevalent deformities, whilst subjects with an $\mathrm{A}+\mathrm{M}$ deformity had a sixfold increase in risk, compared to subjects with no deformity at baseline. There was no evidence that the effects of different shapes of deformity differed between men and women $\left(\chi^{2}=4.1\right.$ on 5 d.f., $P>$ $0.5)$.

\section{Severity of baseline deformity}

The severity of the baseline deformity was measured as the choice of threshold (in standard deviations) for the McCloskey-Kanis algorithm at which that vertebra ceased to be classed as a deformity. This was generally the second most extreme ratio in the vertebra, since a vertebra needs to satisfy two conditions to be classed as a deformity using this method.

If the most severe deformity in the spine had a severity between 3 and $4 \mathrm{SD}$, the odds ratio for an incident fracture was $2.2(95 \%$ CI $1.2,4.2)$. The odds were slightly greater for deformities between 4 and 5 SD $(2.8$ : 95\% CI 1.2, 6.3), and considerably greater for deformities of more than $5 \mathrm{SD}$ (10.9, 95\% CI 7.9, 15.0).

There was a strong association between shape and severity, as shown in Fig. 1. Vertebrae in which the mid height alone, anterior and mid heights, or all three heights were affected tended to be more severe than the other types of deformity.

\section{Risk to adjacent vertebrae}

After adjusting for age, gender, and location, the risk of an incident fracture varied significantly with the distance from the nearest preexisting deformity $\left(\chi^{2}=13.4\right.$ on 4 d.f., $P=0.01$ ); see Table 6 . The presence of a vertebral deformity at baseline increased the risk of an incident fracture sixfold compared to subjects without deformity at baseline, but this risk varied with the distance from the baseline deformity: the risk increased by a factor of 7.7 (95\% CI; 5.6, $10.5)$ within three vertebrae either side of the baseline deformity, and by a factor of $4.0(2.6,6$.) in more distant vertebrae. There was no evidence that the effect of distance from the nearest prevalent deformity differed by vertebral level $\left(\chi^{2}=6.9\right.$ on 12 d.f., $\left.P=0.9\right)$.

\section{Model comparison}

The AIC for each model is presented in Table 7. The models are given in order of increasing AIC: i.e., the best fitting model at the top, the least well-fitting model at the bottom. It is apparent that many of the models have similar AICs: they fit the data equally well. The models including morphometric shape or the number of baseline deformities fit better than those using the other parameters. The best prediction was obtained using the morphometric shape (using the detailed classification). The only other predictor, when added to this model, which led to a significant improvement in fit was severity. However, the improvement in

Table 5

Relative risks of incident vertebral fracture according to shape of baseline deformity

\begin{tabular}{lll}
\hline Type of deformity & Shape & RR $(95 \% \mathrm{CI})^{\mathrm{a}}$ \\
\hline McCloskey-Kanis & Biconcavity & $3.7(2.6,5.3)$ \\
& Wedge & $3.4(2.1,5.5)$ \\
Detailed (see Table 1) & Crush & $4.4(3.1,6.2)$ \\
& M only & $3.3(2.3,4.8)$ \\
& A only & $1.9(1.0,3.4)$ \\
& A + M & $5.9(4.1,8.6)$ \\
& M+P & $1.6(0.8,3.2)$ \\
& A + P & $2.0(0.4,9.5)$ \\
& A + M+P & $3.0(1.8,4.9)$
\end{tabular}

${ }^{\mathrm{a}}$ Relative risk for an incident fracture of any shape associated with a prevalent deformity of the given shape. Referent group is subjects with no prevalent deformities. 


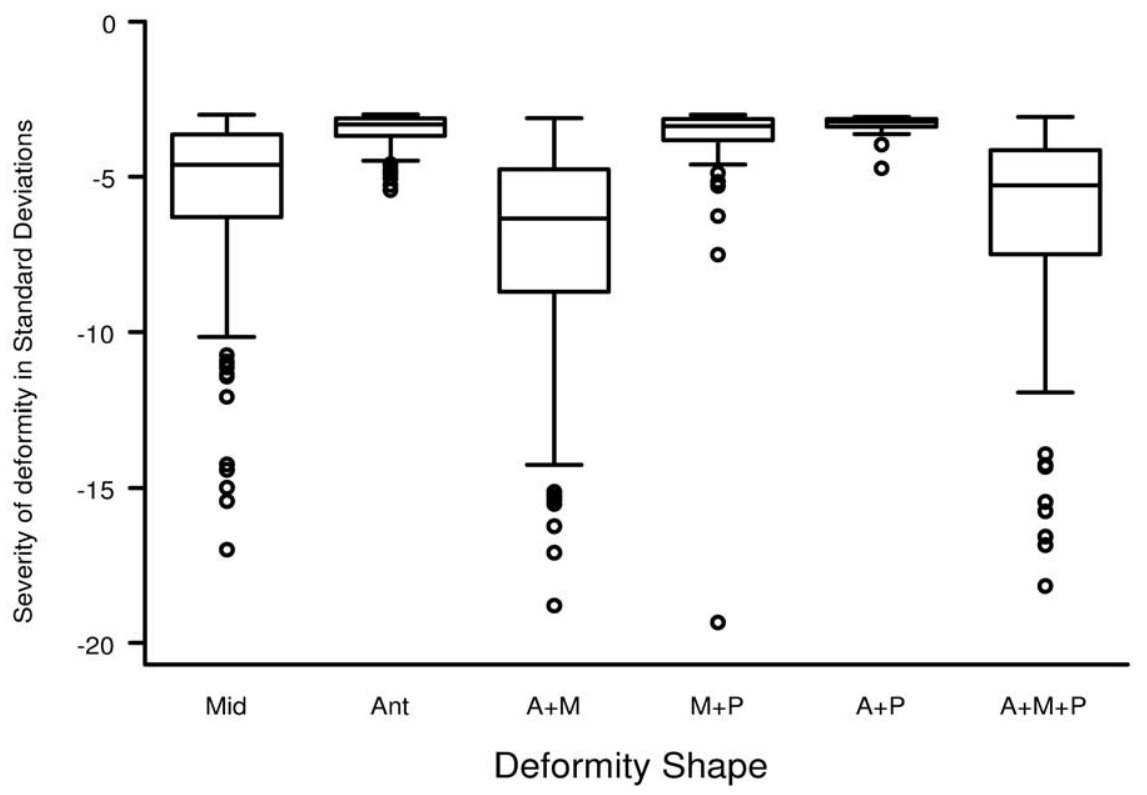

Fig. 1. Distribution of severity of deformity according to deformity shape.

fit when adding severity to the model containing shape $\left(\chi^{2}\right.$ $=13.2$ on 3 d.f.) was considerably less than the improvement in fit when adding shape to the model containing severity (49.3 on 6 d.f.), suggesting that differences in shape capture most of the information given by differences in severity. This combined model had an AIC of 2652, and an AUC of 0.7978 .

\section{Discussion}

Clearly, having a deformity at baseline increases the risk of an incident fracture elsewhere in the spine in both men and women. In this analysis, the risk increased as the number of deformities at baseline increases, and varied according to the shape and severity of the prevalent deformity. The level of risk also varied depending on which vertebral level was affected at baseline, and was greater for those vertebrae anatomically close to the baseline deformity than for verte-

Table 6

Effect of proximity of baseline deformity on incidence of vertebral deformity

\begin{tabular}{lcl}
\hline $\begin{array}{l}\text { Distance in vertebrae to nearest } \\
\text { baseline deformity }\end{array}$ & Relative risk $(95 \% \mathrm{CI})$ \\
\hline Five categories & 1 & $7.3(4.9,10.9)$ \\
& 2 & $7.8(5.1,11.9)$ \\
& 3 & $8.4(5.4,13.1)$ \\
& 4 & $5.9(3.3,10.5)$ \\
Two categories & 5 or more & $3.2(1.9,10.5)$ \\
& $<=3$ & $7.7(5.6,10.5)$ \\
& $>3$ & $4.0(2.6,6.0)$ \\
\hline
\end{tabular}

Note. Referent group is subjects with no prevalent deformities. brae elsewhere in the spine. Apart from shape and severity, these effects were not additive; adding a second predictor to the model did not improve the fit.

This study has the advantage of being large, with nearly 7000 subjects, and included both men and women. In addition, the methods for performing and evaluating both baseline and follow-up radiographs were standardised. There are, though, a number of methodological issues that need to be considered in interpreting the results. The participation rate in the follow-up phase of this study was modest $(50 \%)$. Subjects who did not return for a second $\mathrm{x}$-ray were slightly older and were more likely to have a deformity at baseline than those who took part, which suggests that they may have had a higher rate of incident fracture. In a more detailed analysis using the baseline data, however, such differences appeared to have relatively little impact on incidence [10]. Further, any nonparticipation will only have influenced the results if the association between the various characteristics of baseline deformity and inci-

Table 7

Goodness of fit of prediction models

\begin{tabular}{lll}
\hline & AIC $^{\mathrm{a}}$ & AUC $^{\mathrm{b}}$ \\
\hline Detailed shape classification & 2660 & 0.7969 \\
McCloskey-Kanis shape & 2678 & 0.7972 \\
Number of baseline deformities & 2684 & 0.7973 \\
Severity & 2701 & 0.7960 \\
Location of baseline deformities & 2725 & 0.7946 \\
Nearest baseline deformity (two categories) & 2745 & 0.7911 \\
Nearest baseline deformity (five categories) & 2748 & 0.7917 \\
Presence of a baseline deformity & 2759 & 0.7897 \\
Age, gender, and vertebral level (basic model) & 2935 & 0.7203
\end{tabular}

a Akaike's Information Criterion (see text).

${ }^{\mathrm{b}}$ Area under curve (see text). 
dent fracture differed between those with and without follow-up x-rays, which is unlikely.

In our study we used the vertebra as the unit of analysis. However, the relative risks at the vertebral level are very similar to the relative risks at the subject level, since the risk of an incident fracture in any given vertebra is small. Thus, the relative risk of incident fracture for those having one, two, or more deformities was calculated as 3.1, 9.5, and 21.0, respectively, only slightly less than the per-vertebra risks of 3.2, 9.8, and 23.3.

In the analysis we used a morphometric definition of incident vertebral fracture. Since there is no gold standard for the diagnosis of incident deformities, it is inevitable that any method will be subject to some degree of misclassification. However, any misclassification is unlikely to differ between those with and without prevalent deformities, or between those with baseline deformities of different shapes or in different locations. Its effect would therefore be to reduce the observed association between the predictors and outcome.

Previous studies have found that the existence of a prevalent deformity is a risk factor for an incident fracture [1,20,21]. Two principal mechanisms have been proposed: either that there are local effects of a prevalent deformity, perhaps due to mechanical factors or immobility; alternatively prevalent deformities might be a marker for some other, unmeasured, global risk factor or risk factors affecting the entire spine.

Bone mineral density (BMD) is the most obvious risk factor, but others have shown that prevalent deformities predict incident fractures independently of BMD [1,21]. Although BMD was measured on a subsample of the subjects in this analysis, the numbers were not sufficient to allow the inclusion of BMD in this analysis (where we used the vertebra as the unit of analysis, rather than the person). Bone fragility and propensity to fall are other possible global determinants of risk. The fact that the risk was elevated throughout the spine of subjects with prevalent deformities suggests that such global risk factors were important. However, the fact that the risk was greater in the vertebrae adjacent to a prevalent deformity than elsewhere suggests that local factors also had a significant effect.

The fact that subjects with multiple prevalent deformities are at greater risk of incident fractures is not surprising. These subjects are most likely to have increased global risk factors, such as reduced BMD. In addition, if prevalent deformities increase the risk of subsequent fracture through mechanical influences and immobility, having multiple prevalent deformities will tend to increase the number of vertebrae at elevated risk due to these influences.

The significantly different levels of risk of incident vertebral fracture with different shaped prevalent deformities is a new and interesting observation. There are already data that suggest that BMD is more strongly associated with some shapes of prevalent deformity than others [4]. The shapes of prevalent deformity associated with increased risk of incident fracture in this study tended to be those with the strongest association with low BMD in that study. It has also been suggested that some shapes (for example, reduction only in anterior height) can be caused by other processes, such as degenerative disease [22]. Thus it may be that certain shapes of deformity are less likely to be osteoporotic fractures, being either false positives or deformities due to other causes, and these shapes will be less predictive of future incident fractures. Radiological assessment should therefore take account of both the number of deformities and their shapes.

It is possible to define severity as the sum of the loss of height over the three measured heights in an individual vertebra. However, this means that vertebrae with two affected heights will tend to be classed as more severe than vertebrae with only one affected height, leading to an induced association between severity and shape. We chose instead to define severity as the loss of height of the worst affected height. Even using this definition of severity, however, there were strong correlations between severity and shape; see Fig. 1. The strong correlations make it difficult to separate out the relative contribution of shape and severity to the risk of vertebral fracture. In our analysis, both shape and severity were independent predictors of risk, although shape appeared more important than severity.

The differences in predictive power between prevalent deformities at different vertebral levels are harder to explain. They may be due in part to the fact that the morphometric definition of a prevalent vertebral deformity depends on the standard deviation of the height ratios. If the natural variation in shape at one level (i.e., the standard deviation of the height ratios) is greater than at another, a vertebra will have to be more deformed (i.e., the ratio will have to be reduced to a greater extent) to class as a deformity if it occurs in the first vertebra, rather than in the second. Thus it may be that at some vertebral levels all deformities are detected, whilst at others only more severe deformities are.

A further alternative is that osteoporotic fractures are more common at certain vertebral levels. False positives, on the other hand, should be equally common at all levels, since a particular $z$-score is used to define them. Therefore, the proportion of deformities that are due to fracture will differ between vertebral levels. Since false positives should not be associated with an increased risk of incident vertebral fracture, the strength of the association between prevalent deformity and incident fracture will decrease as the proportion of false positives increase.

There was a slightly greater risk of incident vertebral fracture close to a prevalent deformity than further away. This suggests that local factors are important in influencing risk. The nature of these is unclear, although biomechanical factors are likely to be important. Under normal conditions, 
compressive loads in the spine are directed perpendicular to the vertebral endplate [23]. It seems likely that with progressive deformity, changes in load distribution occur, resulting in an increased susceptibility to fracture for a given BMD.

In a previous study, a prevalent deformity increased the risk of subsequent deformity at vertebrae distant from the initial deformity [20], but that analysis looked only at three distinct regions in the spine, not at individual vertebrae.

It is well established that an individual with a deformity in the spine is at greater risk of subsequent fractures than one with no deformities at baseline. This work has shown that not all deformities increase the risk of subsequent fracture in the same way. Using information about the number, shape, and location of any prevalent deformities may enable better assessment of the risk of future vertebral fractures.

\section{Appendix. Incidence of vertebral fracture}

The table gives the incidence of vertebral fracture per 10,000 vertebrae per year for a woman aged 65 . Multiply the numbers below by 0.45 to obtain the incidence in men, and by 2.2 per decade increase in age to obtain the risk at different ages.

Predicted probabilities of incident fracture by vertebral level (age 65 years, female gender)

\begin{tabular}{lr}
\hline Vertebra & Fractures per 10,000 vertebrae per year \\
\hline T4 & 1.7 \\
T5 & 1.4 \\
T6 & 6.3 \\
T7 & 8.0 \\
T8 & 11.6 \\
T9 & 6.8 \\
T10 & 9.1 \\
T11 & 11.5 \\
T12 & 16.6 \\
L1 & 28.0 \\
L2 & 12.0 \\
L3 & 10.5 \\
L4 & 12.2 \\
\hline
\end{tabular}

\section{References}

[1] Klotzbuecher CM, Ross PD, Landsman PB, Abbott TA III, Berger M. Patients with prior fractures have and increased risk of future fractures: a summary of the literature and statistical synthesis. J Bone Miner Res 2000;15(4):721-39.

[2] Ross PD, Davis JW, Epstein RS, Wasnich RD. Pre-existing fracture and bone mass predict vertebral fracture incidence in women. Ann Intern Med 1991;114(11):919-23.

[3] Ismail AA, Cockerill W, Cooper C, Finn JD, Abendroth K, Parisi G, Banzer D, Benevolenskaya LI, Bhalla AK, Armas JB, Cannata JB, Delmas PD, Dequeker J, Dilsen G, Eastell R, Ershova O, Falch JA, Felsch B, Havelka S, Hoszowski K, Jajic I, Kragl U, Johnell O, Lopez
Vaz A, Lorenc R, Lyritis G, Marchand F, Masaryk P, Matthis C, Miazgowski T, Pols HAP, Poor G, Rapado A, Raspe HH, Reid DM, Reisinger W, Janott J, Scheidt-Nave C, Stepan J, Todd C, Weber K, Woolf AD, Ambrecht G, Gowin W, Felsenberg D, Lunt M, Kanis JA, Reeve J, Silman AJ, O’Neill TW. Prevalent vertebral deformity predicts incident hip though not distal forearm fracture: results from the European Prospective Osteoporosis Study. Osteoporos Int 2001; 12(2):85-90.

[4] Lunt M, Felsenberg D, Reeve J, Benevolenskaya LI, Cannata J, Dequeker J, Dodenhof C, Falch JA, Masaryk P, Pols HAP, Poor G, Reid DM, Scheidt-Nave C, Weber K, Varlow J, Kanis JA, O’Neill TW, Silman AJ. Bone density variation and its effects on risk of vertebral deformity in men and women studied in 13 European centres: the EVOS study. J Bone Miner Res 1997; 12(11):1883-94.

[5] Lee CA, Einhorn TA. The bone organ system: form and function. In: Marcus R, Feldman D, Kelsey J. 2nd ed. Osteoporosis, Vol. 1. San Diego: Academic press, 2001. pp. 3-20.

[6] Melton I, Joseph L, Kan SH, Frye MA, Wahner HW, O'Fallon WM, Riggs BL. Epidemiology of vertebral fractures in women. Am J Epidemiol 1989;129(5):1000-10.

[7] van Staa TP, Dennison EM, Leufkens HGM, Cooper C. Epidemiology of fractures in england and wales. Bone 2001;29(6):517-22.

[8] Ismail AA, Cooper C, Felsenberg D, Varlow J, Kanis JA, Silman AJ, O'Neill TW. Number and type of vertebral deformities: epidemiological characteristics and relation to back pain and height loss. Osteoporos Int 1999;9(3):206-13.

[9] O’Neill TW, Felsenberg D, Varlow J, Cooper C, Kanis JA, Silman AJ, \& the European Vertebral Osteoporosis Study Group. The prevalence of vertebral deformity in European men and women: the European Vertebral Osteoporosis Study. J Bone Miner Res 1996;11: 1010-7.

[10] The European Prospective Osteoporosis (EPOS) Study Group. Incidence of vertebral fracture in Europe: results from the European Prospective Osteoporosis Study (EPOS). J Bone Miner Res 2002; 17(4):716-24.

[11] McCloskey E, Spector TD, Eyres KS, Fern ED, O'Rourke N, Wasikaran S, Kanis JA. The assessment of vertebral deformity: a method for use in population studies and clinical trials. Osteoporos Int 1993;3(3): $138-47$.

[12] Lunt M, Gowin W, Johnell O, Armbrecht G, Felsenberg D, Reeve J. A statistical method to minimise magnification errors in serial vertebral x-rays. Osteoporos Int 2001;12:909-13.

[13] Weber K, Lunt M, Felsenberg D, Lauermann T, Gowin W, Wieland EU, O'Neill TW, Reeve J. Measurement imprecision in digital vertebral morphometry of spinal radiographs in the European Prospective Osteoporosis Study: consequences for the identification of prevalent and incident deformities. Brit J Radiogr 1999;72(862):957-66.

[14] Black DM, Cummings SR, Karpf DB, Cauley JA, Thompson DE, Nevitt MC, Bauer DC, Genant HK, Haskell WL, Marcus R, Ott SM, Torner JC, Quandt SA, Reiss TF, Ensrud KE, \& the Fracture Intervention Trial Research Group. Randomised trial of the effect of alendronate on risk of fracture in women with existing vertebral fractures. Lancet 1996;348:1535-41.

[15] Liang K.-Y, Zeger SL. Longitudinal data analysis using generalized linear models. Biometrika 1986;73(1):13-22.

[16] StataCorp. Stata statistical software: Release 6.0. College Station, TX: Stata Corp.,1999.

[17] Akaike H. A new look at statistical model identification. IEEE Trans Automat Control 1974;AC-19:716-23.

[18] Hanley J, McNeill B. The meaning and use of the area under a receiver operating characteristic (ROC) curve. Radiology 1982;143: 29-36.

[19] Cooper C, Atkinson EJ, O'Fallon WM, Melton LJ 3rd. Incidence of clinically diagnosed vertebral fractures: a population-based study in 
Rochester, Minnesota, 1985-1989. J Bone Miner Res 1992;7(2): 221-7.

[20] Davis JW, Grove JS, Wasnich RD, Ross PD. Spatial relationships between prevalent and incident spine fractures. Bone 1999;24(3): 261-4.

[21] Ross PD, Genant HK, Davis JW, Miller PD, Wasnich RD. Predicting vertebral fracture incidence from prevalent fractures and bone density among non-black, osteoporotic women. Osteoporos Int 1993;3(3): $120-6$.
[22] Abdel-Hamid Osman A, Bassiouni H, Koutri R, Nijs J, Guesens P, Dequeker J. Aging of the thoracic spine: distinction between wedging in osteoarthritis and fracture in osteoporosis-a cross-sectional and longitudinal study. Bone 1994;15(4): 437-42.

[23] Edmondston SJ, Singer KP, Day RE, Price RI, Breidahl PD. Ex vivo estimation of thoracolumbar vertebral body compressive strength: the relative contributions of bone densitometry and vertebral morphometry. Osteoporos Int 1997;7:142-8. 